\title{
The influence of magnetic field curvature on intermittency in drift-wave turbulence in the stellarator $\mathrm{TJ}-\mathrm{K}$
}

\author{
S. Garland, ${ }^{1, \text { a) }}$ P. Manz, ${ }^{2}$ and M. Ramisch ${ }^{1}$ \\ ${ }^{1)}$ Institute of Interfacial Process Engineering and Plasma Technology IGVP, University of Stuttgart, 70569 Stuttgart, \\ Germany \\ 2) Max Planck Institute for Plasma Physics, 85748 Garching, Germany
}

(Dated: 25 May 2020)

The influence of magnetic field curvature on the intermittency in density and potential fluctuations in driftwave turbulence has been investigated in the stellarator TJ-K in the framework of an extended HasegawaWakatani model. A structure function analysis was used to estimate the intermittency level of poloidally resolved drift-wave turbulence measurements of a deuterium plasma in TJ-K. Potential fluctuations were found to be broadly self-similar, whereas density fluctuations were found to be more intermittent in the region with negative normal and positive geodesic curvature. This behaviour could be understood by comparing the data to two-dimensional extended Hasegawa-Wakatani simulations which retain gradients in the magnetic field strength, giving rise to curvature effects. The model is able to reproduce the trends in the experimental data if both normal and geodesic curvature effects are accounted for, as well as the local anisotropy of turbulent length scales. The analysis indicates the importance of local magnetic geometry as a factor in the decoupling of density and potential fluctuations, leading to intermittency in drift-wave turbulence.

Keywords: Fusion, Magnetic confinement, Intermittency, Hasegawa-Wakatani, Stellarator

\section{INTRODUCTION}

The transport of particles and heat by turbulent eddies from the edge region of magnetically confined plasmas towards the reactor wall lowers the confinement time and poses a threat to plasma facing components ${ }^{1,2}$. A large proportion of this transport is often attributed to long-lived plasma filaments, characterised by intermittent fluctuations in the plasma pressure, known as blob filaments ${ }^{3,4}$. The effect of magnetic field curvature on the dynamics of these filaments is well documented ${ }^{5-7}$, and there is evidence that it can localise blob generation ${ }^{8}$. The paradigm for blob generation is a shearing-off of high amplitude drift waves in the flow-shear region at the plasma edge $\mathrm{e}^{9,10}$. Therefore, the statistical properties relating to intermittency in this region, dominated by drift-wave turbulence, are of interest regarding the properties of turbulent transport in the scrape-off layer (SOL).

In the adiabatic limit of drift-wave turbulence, where the collisionality is very low, the density field is strongly coupled to the electrostatic potential resulting in electrically unpolarised density perturbations. A previous study $^{11}$ has shown that a decoupling of density and potential in drift-wave turbulence via increased collisionality leads to intermittency in the density fluctuations. It is thus reasonable to assume that a magnetic field curvature driven polarisation of density perturbations could have a similar effect on the intermittency of the turbulence.

\footnotetext{
a) Corresponding author.

Current address: Deutches Zentrum für Luft- und Raumfahrt, Rutherfordstraße 2, 12489 Berlin

Email: stephen.garland@dlr.de
}

Indeed a study has shown the importance of the normal component of the magnetic field curvature in determining intermittency in drift-wave turbulence in simulations ${ }^{12}$, however experimental evidence is still lacking.

The experiment TJ-K $\mathrm{K}^{13}$ is ideally suited for detailed comparison with simulation results. Low plasma densities and temperatures allow Langmuir probe access in the entire plasma volume. Arrays of probes can be used to investigate the spatial variation of the properties of edge turbulence and compare them to local parameters such as magnetic field curvature.

The Hasegawa-Wakatani model ${ }^{14,15}$ for drift-wave turbulence provides a relatively simple framework within which to interpret the experimental data in terms of the underlying physical mechanisms. The model may be extended to introduce the effect of an inhomogeneous magnetic field, without modifying the simulation grid geometry, in order to reproduce the effect of magnetic curvature ${ }^{16}$. The resulting extended HasegawaWakatani (EHW) model predicts a difference in the intermittency of drift-wave turbulence between the good and bad-curvature regions in magnetic confinement fusion devices ${ }^{12}$.

The paper is laid out as follows. In section II the structure function method of analysing intermittency is presented. Following this, in section III details of the stellarator experiment TJ-K are given, including the diagnostics required for poloidally resolved fluctuation measurements. The main experimental results are then presented in section IV. The EHW model is described in section $\mathrm{V}$, following Dura et $\mathrm{al}^{12}$ but including a "geodesic curvature" component. In this section, simulations carried out with the model are compared to the experimental data. Finally section VI draws together the important points discussed in sections IV and V. 


\section{CHARACTERISING INTERMITTENCY}

A central assumption of Kolmogorov's K41 three dimensional turbulence model ${ }^{17,18}$ and Kraichnan's Kr67 model $^{19,20}$ for two dimensional turbulence is self-similar motion in the inertial ranges of the turbulence spectrum. Self-similar turbulence implies no prefered direction of flow and quasi-Gaussian statistics across all scales involved in the inertial range. A lack of selfsimilarity implies intermittency, which can essentially be thought of as a scale-variance of the statistical moments of the probability density function. Several methods exist to characterise the intermittent nature of datasets (see for example ${ }^{18,21}$ ), however a commonly used method is that of structure function analysis (see for example Refs. $\left.{ }^{18,22,23}\right)$. The structure function of order $q$ for a spatially varying quantity $X(\mathbf{r})$ is calculated from

$$
S_{q}(d)=\left\langle|X(\mathbf{r}+d)-X(\mathbf{r})|^{q}\right\rangle=\left\langle\left|\delta X_{d}(\mathbf{r})\right|^{q}\right\rangle
$$

where $d$ is the displacement between points in space and $\langle\ldots\rangle$ represents an average over space. The absolute value of $\delta X_{d}(\mathbf{r})$ is taken to improve convergence ${ }^{24,25}$. The $q$ th order structure function is the statistically averaged $q$ th moment of the distribution of the difference variable $\delta X$ as a function of separation $d$. The second order structure function, for example, corresponds to the covariance of the difference functions at points $\mathbf{r}$ and $\mathbf{r}+d$. In the field of neutral fluids, intermittency in the velocity field is commonly studied and so $X$ is the velocity $v$ and $\delta v=(v(\mathbf{r}+d)-v(\mathbf{r}))$. In the case of plasma turbulence density perturbations can actively influence the flow dynamics, which is in contrast to neutral fluids where the density is only passively advected. Thus in plasmas the density as well as the vorticity are of interest, besides the flow field itself ${ }^{12,24,26}$. The scaling of the structure functions as a function of the displacement $d$ between two points provides information on scale-invariance. The analysis proceeds by looking for a power-law relation of the form $S_{q} \propto d^{\zeta_{q}}$, to extract the scaling exponent $\zeta_{q}$. If $\zeta_{q}$ is a linear function of $q$ then the data is said to be self-similar, however, a deviation from a linear relationship indicates the presence of intermittency. The fitting of a power-law relation to the structure functions is greatly facilitated by the extended self-similarity (ESS) technique $^{27}$, which consists of plotting all structure functions against $S_{3}$, thereby reducing the impact of undetermined undulations in the raw structure function data ${ }^{18}$. The third order structure function is used in the ESS analysis due to Kolmologrov's four-fifths law, which implies the property $\zeta_{3}=1$ (see Ref. 18). Thus the form of $\zeta_{q}$ is not being altered through this normalisation. Scaling exponents can then be calculated more accurately from the slope of the ESS plot. To characterise the level of intermittency, allowing comparison between different data sets, the difference between the calculated 6 th order normalised structure function and its non-intermittent prediction of 2 can be taken $^{18,22,23,28}, \mu \equiv 2-\zeta_{6} / \zeta_{3}$.
This quantity will be used in order to compare the intermittency level at different poloidal positions. The 6 th order structure function was first introduced as a fitting parameter in the log-normal intermittency model ${ }^{18}$ and, for comparison, it is commonly used in the literature to characterise the intermittency in terms of the intermittency parameter (see e.g. references 22 and 29). Moreover, the 6th was the highest accessible order, resulting in a smaller percentage uncertainty on the intermittency parameter.

Experimentally, spatial variations in the potential and electron density fields are difficult to access with sufficient resolution. However, if the turbulent flow fluctuations are treated as small compared to the equilibrium flow (Taylor's hypothesis ${ }^{30}$ ) then the spatial structure, frozen in the flow, maps to temporal variations for measurements taken at a fixed position. The corresponding structure functions are then calculated in time using $S_{q}(\tau)=\left\langle|\delta x(t)|^{q}\right\rangle$, with a time lag between points of $\tau$. Plasma potential measurements in TJ-K have shown that the fluctuating $E \times B$ velocity is typically a factor of 3-4 times smaller than mean flow at the inner edge of the separatrix, indicating the validity of the Taylor hypothesis.

\section{EXPERIMENTAL SETUP}

Experiments were carried out at the stellarator TJ$\mathrm{K}^{13}$, depicted schematically in figure $\left.1 \mathrm{a}\right)$. TJ-K is a small sized $l=1$ stellarator (torsatron) with a major radius of $R=0.6 \mathrm{~m}$ and a minor radius of approximately $a=0.1 \mathrm{~m}$. The single helical coil has a 6 -fold symmetry in the toroidal coordinate. Plasma breakdown is achieved with electron cyclotron resonant heating using a $2.45 \mathrm{GHz}$ magnetron with up to $3 \mathrm{~kW}$ heating power ( $\mathrm{see}^{31}$ for details on plasma heating in TJ$\mathrm{K})$. For the deuterium plasmas generated for these experiments, peak electron densities are typically of the order of $n_{\mathrm{e}} \approx 5 \times 10^{17} \mathrm{~m}^{-3}$, whilst electron temperatures are around $T_{\mathrm{e}} \approx 10 \mathrm{eV}$ and ions are cold, $T_{\mathrm{i}}<1 \mathrm{eV}$ 32 . Due to the low density and temperature, Langmuir probes can be used in the entire plasma volume without risking damage, enabling large data-sets to be obtained with relatively high spatial resolution. Edge turbulence in TJ-K has been found to be drift-wave dominated ${ }^{33}$. Despite relatively low densities and temperatures the dimensionless quantities relevant to drift-wave turbulence are similar in TJ-K to those in the edge region of larger reactors ${ }^{34}$, making the results obtained here relevant to the fusion community as a whole.

The 2D probe manipulator unit is used to obtain radial profiles of the ion saturation current, $I_{\mathrm{i}, \text { sat }}$, and the electron temperature, $T_{\mathrm{e}}$, through fitting of current-voltage characteristics. These measurements are used in conjunction with a $64 \mathrm{GHz}$ interferometer, measuring the line-averaged electron density, to determine the absolute density values (see Ref. 35 for details) and therefore 

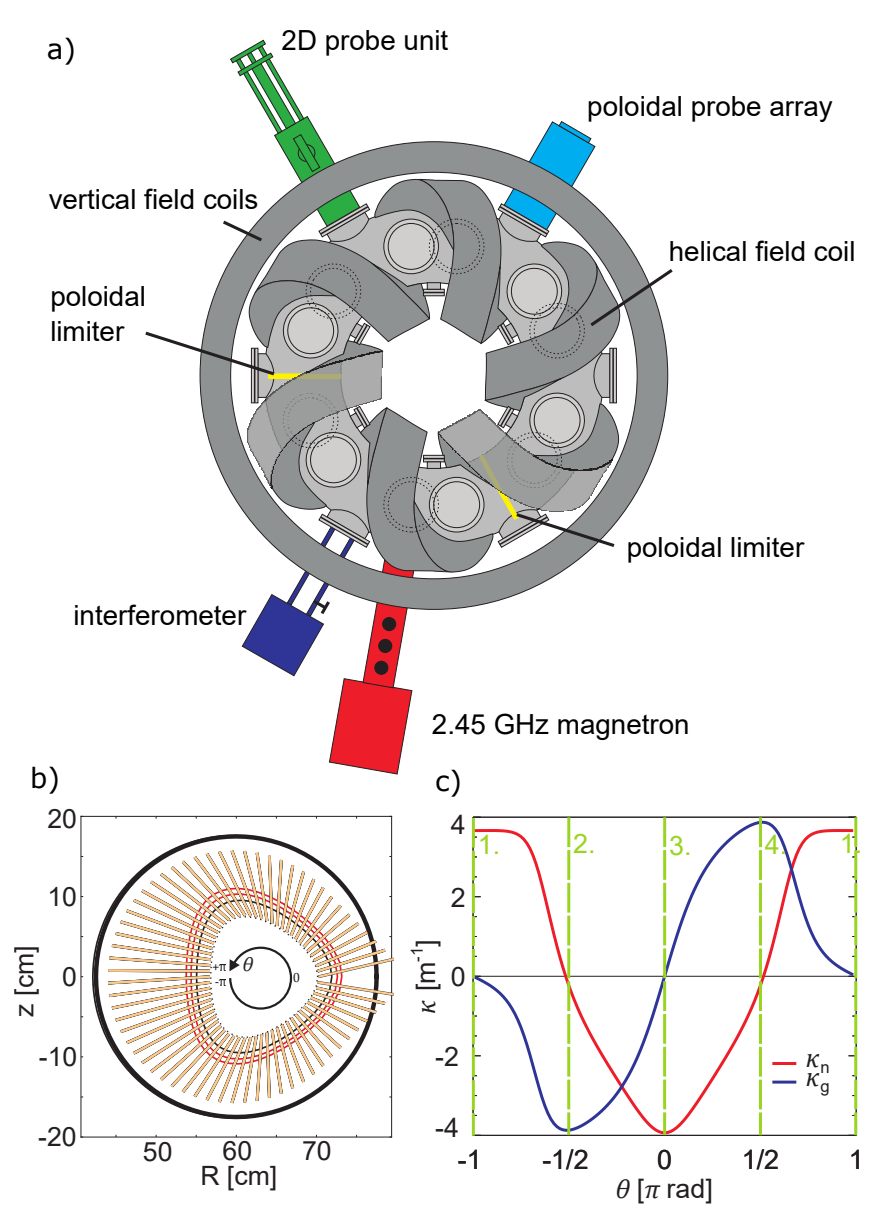

FIG. 1. a) A schematic top view of the stellarator TJ-K with its key diagnostics. b) A poloidal cross-section of the vacuum vessel showing the layout of the outer-port poloidal probe array. c) Normal, $\kappa_{\mathrm{n}}$, and geodesic, $\kappa_{\mathrm{g}}$, curvature as a function of poloidal angle $\theta$ at the array position in TJ-K. Dashed lines and numbering represent curvature parameters taken for local simulations.

the plasma collisionality, $C$. In the case where electronelectron collisions dominate the electron parallel mobility calculated $C$ may be calculated from ${ }^{15}$

$$
\begin{aligned}
C & =\frac{\nu_{\mathrm{ee}} / \omega_{\mathrm{ce}}}{\left(k_{\|} \rho_{\mathrm{s}}\right)^{2}} \\
& =\frac{n_{\mathrm{e}} m_{\mathrm{e}} B e}{1.4 \times 10^{10} m_{\mathrm{i}} k_{\|}^{2} T_{\mathrm{e}}^{5 / 2}},
\end{aligned}
$$

where $\nu_{\mathrm{ee}}$ is the electron-electron collision frequency, $\omega_{\mathrm{ce}}=e B / m_{\mathrm{e}}$ is the electron cyclotron frequency, and $\rho_{\mathrm{s}}$ is the drift scale. Electron and ion masses are given by $m_{\mathrm{e}}$ and $m_{\mathrm{i}}$, and $k_{\|}$is the typical parallel wavenumber of the drift-wave turbulence. The parallel wave number used here for hydrogen was previously determined in Ref. 36. In addition, a pair of poloidal limiters were used (see figure 1a) for location), consisting of a steel plate with the precise shape of flux surface cut into it, ensuring a well defined last closed flux surface (LCFS).

The relationship between magnetic curvature and intermittency can be studied by exploiting the poloidal variation in the normal and geodesic magnetic curvature components, $\kappa_{\mathrm{n}}$ and $\kappa_{\mathrm{g}}$, in TJ-K, depicted in figure 1c). The normal curvature is positive on the inboard side and negative on the outboard side, corresponding to good and bad curvature regions respectively. The geodesic curvature, on the other hand, has an up-down asymmetry with positive values above the mid-plane and negative values below. To this end a poloidal Langmuirprobe array, figure $1 \mathrm{~b}$ ) was used to measure fluctuations in $I_{\mathrm{i}, \mathrm{sat}}$ and $\phi_{\mathrm{fl}}$ at discrete locations on a flux surface. The array consists of 64 equidistant Langmuir probes positioned $8.1 \mathrm{~mm}$ apart located on the intersection between the poloidal measuring plane and a flux surface approximately $1.5 \mathrm{~cm}$ inside the LCFS. Individual probes are constructed from a $0.1 \mathrm{~mm}$ tungsten wire electrically isolated from the plasma with an ceramic aluminium oxide tube, covering the entire tungsten wire apart from a $3 \mathrm{~mm}$ exposed tip. The 64 probes making up the array are secured to a metal ring, ensuring precise positioning of the probe tips along the desired flux surface projection, the coordinates of which were obtained using a field-line tracing code. Consecutive measurements of $\phi_{\mathrm{fl}}$ and then $I_{\mathrm{i} \text {,sat }}$ of $0.5 \mathrm{~s}$ duration at a sampling rate of $1 \mathrm{MHz}$ were obtained simultaneously with all 64 probes, providing long enough data series to calculate up to the 6 th order structure function. Due to a lack of coherent temperature fluctuations in $\mathrm{TJ}-\mathrm{K}^{37}, I_{\mathrm{i}, \text { sat }}$ fluctuations are proportional to those of the electron density, and $\phi_{\mathrm{fl}}$ fluctuations to those of the plasma potential.

\section{EXPERIMENTAL RESULTS}

Measurements of the electron density and potential potential fluctuations (proportional to $I_{\mathrm{i}, \text { sat }}$ and $\phi_{\mathrm{ff}}$ respectively) were performed as a function of poloidal angle using the 64-probe array detailed in section III. From these measurements structure functions were calculated, and an ESS analysis was performed to obtain the intermittency parameter, $\mu=2-\zeta_{6} / \zeta_{3}$, as detailed in section II. Example structure functions up to order $q=6$ are shown in figure 2 a) for a probe at the low field side of the probe array, at $\theta=0 \mathrm{rad}$. The structure functions have similar forms to those found for ion saturation current measurements in other edge plasmas ${ }^{38}$. The aim of the structure function intermittency analysis is to look for a power law scaling with the time lag, $S_{q} \propto \tau^{\zeta_{q}}$. A power-law scaling region (linear on the log-log scale) in Fig. 2a) is not obvious, making the accurate estimation of $\zeta_{q}$ difficult. The ESS technique, however exhibits a much larger region of power law scaling, as can be seen from Fig. 2b), allowing a more accurate estimation of the scaling exponent. The quantity of data was sufficient to allow ESS analysis of the 6th order structure function 
a)

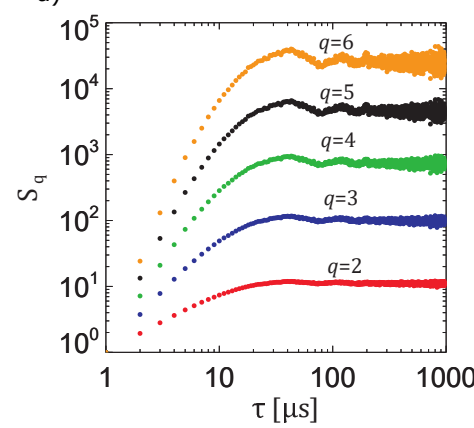

c)

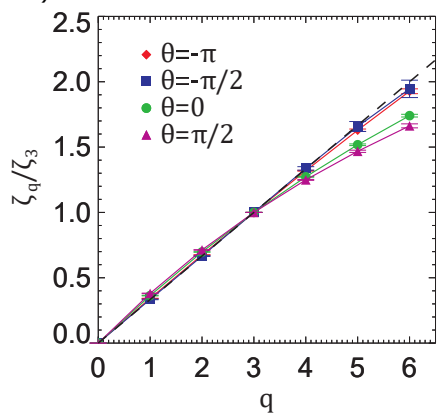

b)

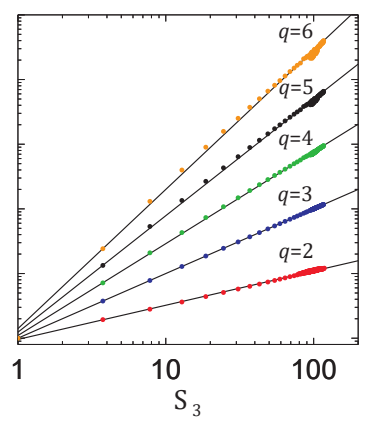

FIG. 2. a) Example structure functions from $I_{\mathrm{i}, \text { sat }}$ measurements at the mid-plane probe on the outboard side. b) ESS analysis of the same data along with linear fits (black lines). c) Scaling of power-law exponent $\zeta_{q} / \zeta_{3}$ with order $q$.

exponents of $I_{\mathrm{i} \text {,sat }}$ signals at all poloidal locations. Due to the length of the time series $(\approx 0.5 \mathrm{~s})$, the maximum structure function order was limited to $q=6$. This limit was determined following the analysis in Ref. 39. The resulting scaling curves from this analysis are shown for 4 poloidal positions in Fig. 2c).

Fig. 3 shows the result of the ESS analysis repeated for all 64 probes in the array, leading to the intermittency parameter, $\mu$, for density and potential fluctuations as a function of poloidal angle. From this it can be seen that the potential fluctuations have low $\mu(<0.1)$ across the range of poloidal angles, indicating self-similarity. This is consistent with other observations of the plasma potential $^{12,24,29,40}$. The density on the other hand exhibits more intermittent behaviour, signified by a higher $\mu$, in the region $0<\theta<3 \pi / 4$. In general, there is a peak in the intermittency parameter on the low-field side. This can be compared to previous predictions of more intermittent fluctuations on the low-field side $^{12}$, which can be explained due to the normal component of the magnetic curvature. In the measurements shown in Fig. 3 the intermittency parameter profile is shifted compared to what would be expected from a model containing only the normal curvature component. In section $\mathrm{V}$ it will be shown that it is possible to explain the form of the profile by introducing a geodesic curvature component and taking into account the anisotropy of the turbulence wavenumber spectrum.

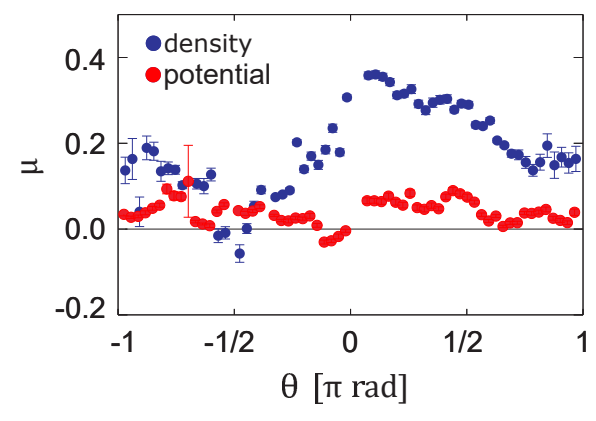

FIG. 3. Poloidal profiles of the intermittency parameter, $\quad \mu$, for density and potential fluctuations measured with the poloidal probe array for a deuterium discharge.

\section{EXTENDED HASEGAWA-WAKATANI SIMULATIONS}

The 2D Hasegawa-Wakatani model ${ }^{14,15}$ captures the essence of drift-wave turbulence in a small number of parameters: $C$ the collisionality, which accounts for dynamics parallel to the magnetic field; and $L_{n}$ the density gradient length scale. The model has been widely used to study fundamental aspects of drift-wave turbulence in relation to its statistical and intermittency properties (see for example ${ }^{12,41-43}$ ). The model can be extended to include an inhomogenous magnetic field whilst retaining the ease of computation of two fields in two dimensions. This is referred to as the Extended Hasegawa-Wakatani (EHW) model ${ }^{16,44,45}$. The equations are based on the Hasegawa-Wakatani model, but include extra terms due to the fact that the divergence of the $E \times B$ and diamagnetic drift velocities no longer vanish when gradients in the magnetic field strength are retained. In this case the equations become

$$
\begin{aligned}
\partial_{t} n+\{\phi, n\}+\partial_{y} \phi \frac{1}{L_{n}} & =\frac{1}{C}(\phi-n)-2\{\ln B, \phi-n\} \\
\partial_{t} \Omega+\{\phi, \Omega\} & =\frac{1}{C}(\phi-n)-2\{\ln B,-n\},
\end{aligned}
$$

where $n$ is the density, $\phi$ the potential, $\Omega=\nabla^{2} \phi$ the vorticity, $L_{n}$ the density gradient length, and $C$ is the collisionality. The Poisson bracket is defined as $\{A, B\}=$ $\partial_{x} A \partial_{y} B-\partial_{x} B \partial_{y} A$. The effect of a non-linear magnetic field is captured by the second term on the right hand side of each equation, where the local magnetic field strength is given by $B$. The model assumes that the pressure gradient is governed by a constant density gradient, $1 / L_{n}$, with $T_{e}=$ const, whilst ions are assumed to be cold with $T_{i} \ll T_{e}$. 2D slab geometry is obtained by taking a characteristic parallel wavenumber for the turbulence, allowing resistive coupling of $n$ and $\phi$ to be modelled by the collisionality factor, $C$.

Simulations have been performed by Dura et $\mathrm{al}^{12}$ with an extended HW model, retaining a constant gradient of the magnetic field in the $x$ coordinate, equivalent to modelling the normal component of the curvature when considering the mid-plane of a tokamak. In this case a value 


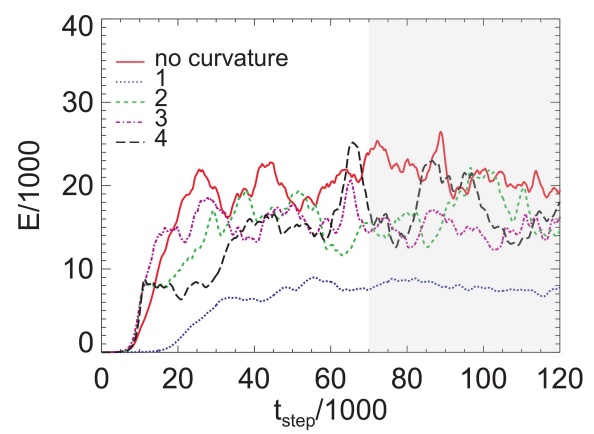

of $\partial \ln B / \partial x$ with the same sign as the density gradient $1 / L_{n}$ corresponds to the region of good curvature, and a value of $\partial \ln B / \partial x$ with the opposite sign corresponds to the region of bad curvature. In addition to a gradient in the magnetic field strength in the $x$ direction, a gradient in the $y$ direction may also be retained from the Poisson bracket, which can play the role of geodesic curvature. To aid a comparison with experimental data, the magnetic gradient in the $x$ and $y$ directions is henceforth referred to as the normal and geodesic curvature respectively, so that we have $\kappa_{n}=\partial \ln B / \partial x$ and $\kappa_{g}=\partial \ln B / \partial y$, although it should be kept in mind that the extended HasegawaWakatani model is still two-dimensional. Making substitutions for $\kappa_{n}$ and $\kappa_{g}$, the EHW equations read

$$
\begin{aligned}
\partial_{t} n+\{\phi, n\}+\partial_{y} \phi \frac{1}{L_{n}}=\frac{1}{C}(\phi-n) & -2 \kappa_{\mathrm{n}} \partial_{y}(\phi-n) \\
& +2 \kappa_{\mathrm{g}} \partial_{x}(\phi-n) \\
\partial_{t} \Omega+\{\phi, \Omega\}=\frac{1}{C}(\phi-n) & -2 \kappa_{\mathrm{n}} \partial_{y}(-n) \\
& +2 \kappa_{\mathrm{g}} \partial_{x}(-n) .
\end{aligned}
$$

For numerical stability, artificial damping terms of the form $\nu \nabla^{2} n$ and $\mu \nabla^{2} \Omega$ (Newton viscosity) were added to the right hand side of equations 3 and 4 , with $\nu=$ $\mu=0.01$, damping high wavenumbers and providing an energy and enstrophy sink. The collisionality parameter $C$ describes the parallel motion of the electrons and was deduced from experimental parameters for the TJ-K case as in Ref. 11.

Local simulations were performed using the EHW model at the four poloidal positions indicated in Fig. 1c) and summarised in table I. The magnitude of $\kappa$ at these locations is calculated by making the curvatures in TJ-K dimensionless via the parameter $\rho_{\mathrm{s}}$. Taking a normalisation factor of $\rho_{\mathrm{s}}$ on the order of $1 \mathrm{~cm}$ the resulting $|\kappa|$ is of the order of $10^{-2}$ to $10^{-1}$ for the local simulation

\begin{tabular}{c|c|c|c|c} 
number & poloidal angle $[\mathrm{rad}]$ & location & $\kappa_{n}$ & $\kappa_{g}$ \\
\hline 1 & $\pm \pi$ & high-field side & 0.1 & 0 \\
\hline 2 & $-\pi / 2$ & bottom & 0 & -0.1 \\
\hline 3 & 0 & low-field side & -0.1 & 0 \\
\hline 4 & $\pi / 2$ & top & 0 & 0.1
\end{tabular}

TABLE I. The curvature characteristics in TJ-K at the 4 discrete locations used in simulations.
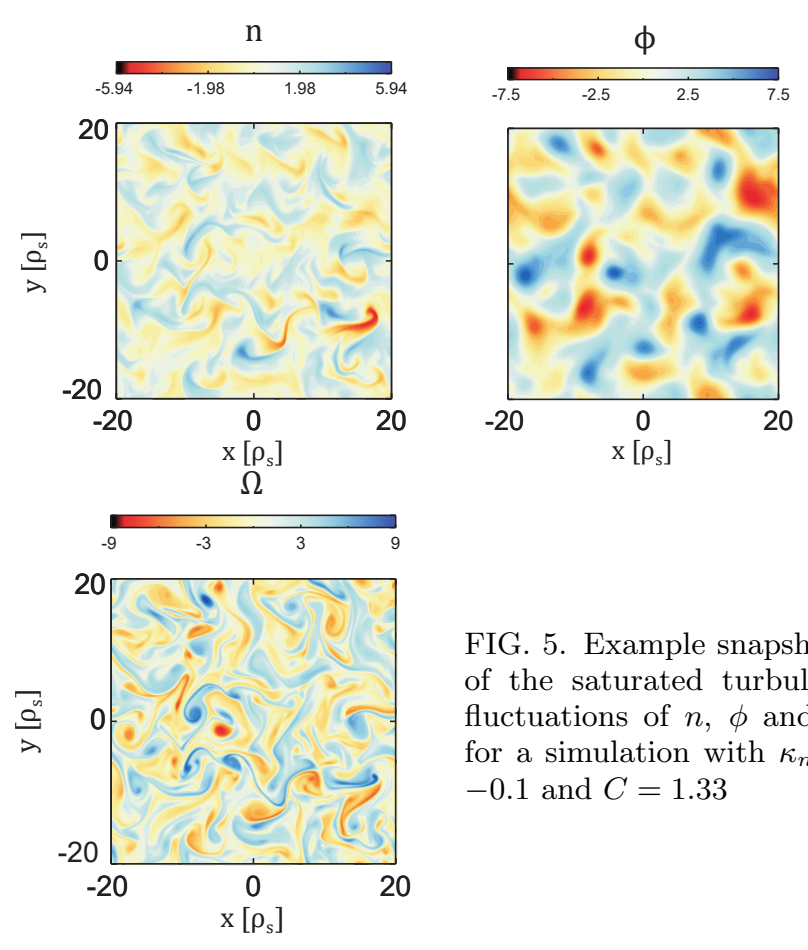

FIG. 5. Example snapshots of the saturated turbulent fluctuations of $n, \phi$ and $\Omega$ for a simulation with $\kappa_{n}=$ -0.1 and $C=1.33$

regions. The collisionality was set at $C=1.33$ in order to compare with the experimental data. All simulations were performed on a $40 \times 40 \rho_{\mathrm{s}}$ grid with $512 \times 512$ nodes, with a time step of $0.005 \rho_{\mathrm{s}} / c_{\mathrm{s}}$, where $c_{\mathrm{s}}=\sqrt{T_{\mathrm{e}} / m_{\mathrm{i}}}$ is the ion speed of sound. Double periodic boundary conditions are used for simplicity, disregarding radial variations in the density profile.

Time-traces of the energy integrated over the simulation grid for each of the four simulations is shown in figure 4. After a growth period of 10000 to 50000 time steps, the energy saturates and a turbulent steady state is reached, following which the statistical properties of simulation data could be analysed. Example snapshots of the $n, \phi$ and $\Omega$ fields for the case with negative normal curvature are shown in figure 5 after a saturation in the integrated energy and enstrophy has been reached. Based on the analysis in Ref. 11, visual inspection of the density field can already yield information on the intermittency of the fluctuations. In this case the density field quite closely resembles the vorticity field, indicating a decoupling of the density and potential fields, and the intermittency of the density. This intermittency can be quantified with a structure function analysis.

A spatial structure function analysis was carried out 
a)

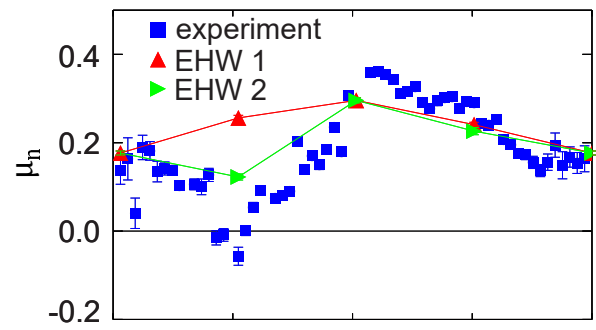

b)

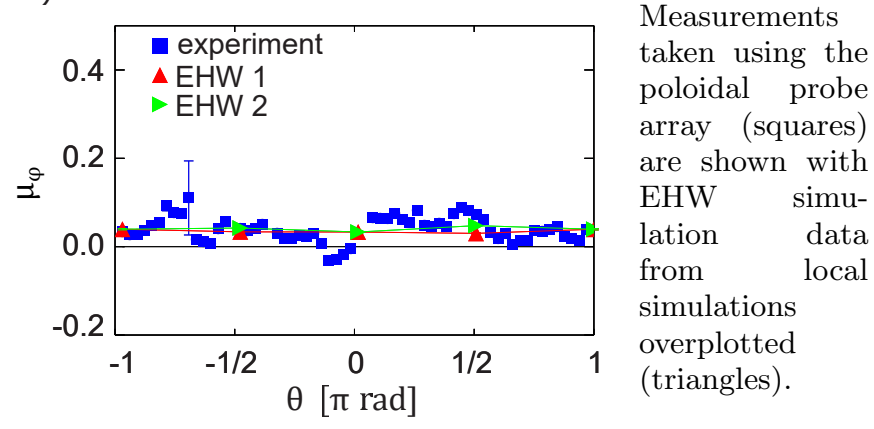

for snapshots of the turbulent fields $n$ and $\phi$ over a period of 50000 time steps (indicated by the shaded region in figure 4 ). The eddy turnover time, $t_{e}$, was between 70 and 140 time steps, where $t_{e}$ is calculated from $t_{e}=1 / W$, and $W$ is rms vorticity ${ }^{43}$. Snapshots were taken equally spaced over 500 time steps (corresponding to $\approx 3-7 t_{e}$ ) in order to obtain better statistics, ensuring that subsequent snapshots are not strongly correlated. As with the experimental data, the intermittency parameter, $\mu$, was obtained through an ESS analysis of the structure functions. The resulting poloidal profile of $\mu$ is shown, labelled as EHW 1, along with the experimental data from section IV in figure 6a) for the density field, $\mu_{n}$, and in figure $6 \mathrm{~b}$ ) for the potential, $\mu_{\phi}$.. As in the experiments, the intermittency parameter for the potential is consistently low over the entire range of $\theta$, indicating self-similar fluctuations in the potential, which matches observations in other studies ${ }^{12,24,29,40}$. The broad trend of $\mu(\theta)$ found in the experimental density fluctuations is reproduced in the simulations above the mid-plane $(0<\theta<\pi)$, however below the mid-plane a large discrepancy can be observed between $-\pi<\theta<0$. In order to understand this discrepancy, and indeed the general form of the profile in $\mu$ for the experimental data, further simulations were carried out to account for spatial inhomogeneity in the turbulence.

The main feature in the EHW 1 simulation poloidal $\mu_{n}$ profile is a higher intermittency parameter on the low-field side, where $\kappa_{\mathrm{n}}<0$ as compared to at $\theta=\pi$, where $\kappa_{\mathrm{n}}>0$. This can be explained by the decoupling of the density and potential fluctuations through the curvature terms in equations 6 when the normal curvature is negative, in a similar way to which the density-potential coupling is influenced by the collisionality parameter in the classic HW model ${ }^{11,12}$. However, the coupling terms in the EHW model, $\kappa_{\mathrm{n}} \partial_{\mathrm{y}}(\phi-n)$ and $\kappa_{\mathrm{g}} \partial_{\mathrm{x}}(\phi-n)$, in-

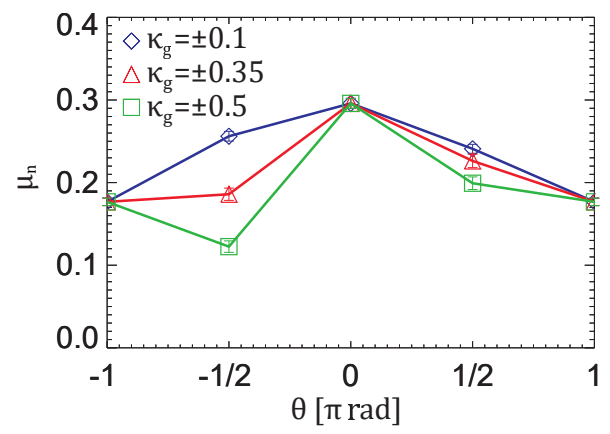

volve taking the spatial gradients $\partial_{\mathrm{x}}$ and $\partial_{\mathrm{y}}$ of the term $(\phi-n)$. Thus in the EHW model the shape of eddies is important. In a previous investigation ${ }^{46}$, a shift of the turbulent transport maximum from the outboard midplane to the region with negative normal and positive geodesic curvature $(\theta \approx \pi / 2)$ could be explained using a linear model of drift-wave growth rates ${ }^{47,48}$. According to this model the drift-wave growth rate was given by

$$
\gamma \propto-\kappa_{\mathrm{n}}+\kappa_{\mathrm{g}} \frac{\chi^{\prime}}{B} G^{s s}\left(\Theta_{k}+\Lambda\right),
$$

where $\chi^{\prime}$ is radial derivative of the poloidal magnetic flux, $\Lambda$ is the integrated local magnetic shear, $\Theta_{k}$ reflects the ratio of the radial and poloidal wavenumbers, and $G^{s s}$ is the metric coefficient in the radial coordinate. This model predicts a shift in the maximum growth rate from the outboard mid-plane (where $\kappa_{\mathrm{n}}$ is most negative) to the region where $\kappa_{\mathrm{n}}<0$ and $\kappa_{\mathrm{g}}>0$. It was found that the characteristics of the experimental poloidal profiles of turbulent radial transport were reproduced best when the ratio of radial to poloidal wavenumbers is approximately 2, corresponding to radial length scales which are shorter than poloidal length scales. Such an effect can be incorporated into the simulations by altering the magnitudes of the curvature terms, since these affect the impact that the curvature has on the density-potential coupling. Thus an impression of the effect of anisotropic length scales in the simulations can be gained by altering the curvature values at different positions.

Figure 7 shows the results of the local EHW simulations with increasing absolute values of the geodesic curvature parameter, reflecting a larger value of the ratio of radial and poloidal wavenumbers. The local simulations incorporating normal curvature (at the poloidal locations $\theta=0, \pm \pi)$ remain the same for the three profiles, with $\kappa_{n}= \pm 0.1$. From the figure it can be seen that increasing $\left|\kappa_{\mathrm{g}}\right|$ results in a decreased $\mu_{n}$ for both positive and negative geodesic curvature regions, however the decrease in $\mu_{n}$ is larger in the region of negative geodesic curvature compared to the region of positive geodesic curvature. Whilst such an analysis is qualitative in nature, it is still interesting to compare the results with the experimental data. The local simulations with $\left|\kappa_{\mathrm{g}}\right|=0.35$ are shown in figure 6 as EHW 2. In the case of the potential field, the profile remains the same, however for the density field, 
the dip in $\mu$ is now reproduced in the simulations. This indicates that the dominant turbulence length scales also play a role in determining where magnetic field curvature leads to density-potential decoupling, and the resulting locally enhanced intermittency levels. A more detailed understanding of this phenomenon would required more complex simulations, taking into account magnetic shear (which also enters into Eq. 7), and in the best case, the full global magnetic field geometry. This is outside of the scope of this paper and is left for future work.

\section{SUMMARY AND CONCLUSIONS}

Poloidally resolved measurements of the intermittency level of drift-wave density and potential fluctuations have been studied in the framework of an extended HasegawaWakatani model. It has been observed that the potential fluctuations are consistently self-similar at all poloidal positions, characterised by a low intermittency parameter, $\mu$. The density fluctuations, on the other hand, exhibit a peak in the intermittency parameter in the region where the normal curvature is negative and the geodesic curvature is positive.

To understand the form of this intermittency profile, complementary two-dimensional extended HasegawaWakatani simulations were performed, retaining gradients in the magnetic field in order to simulate magnetic field curvature. Four local simulations were carried out with local curvature characteristics corresponding to the inboard, outboard, top and bottom of a poloidal cross section. Simulations with these characteristics exhibited a peak in $\mu$ at the outboard mid-plane, where normal curvature is at a minimum and there is no geodesic component of the curvature. This is in contrast to the experimental data, where the peak is shifted into the region with positive geodesic curvature. This shift could be understood by accounting for the anisotropy of the radial and poloidal length scales of drift wave eddies, found in previous studies. By performing simulations with modified geodesic curvature values to account for the larger poloidal compared to radial length scales, the shift of the profile peak away from the mid-plane into the region of positive geodesic curvature could be reproduced.

These findings show the importance of considering local curvature properties as a factor in the decoupling of density and potential fluctuations in drift-wave turbulence, giving rise to intermittent density fluctuations.

\section{DATA AVAILABILITY STATEMENT}

The data that support the findings of this study are available from the corresponding author upon reasonable request.

\footnotetext{
${ }^{1}$ G. Birkenmeier, P. Manz, D. Carralero, F. M. Laggner, G. Fuchert, K. Krieger, H. Maier, F. Reimold, K. Schmid,
}

R. Dux, T. Pütterich, M. Willensdorfer, E. Wolfrum, and The ASDEX Upgrade Team, Nuclear Fusion 55, 033018 (2015).

${ }^{2}$ B. Lipschultz, X. Bonnin, G. Counsell, A. Kallenbach, A. Kukushkin, K. Krieger, A. Leonard, A. Loarte, R. Neu, R. Pitts, T. Rognlien, J. Roth, C. Skinner, J. Terry, E. Tsitrone, D. Whyte, S. Zweben, N. Asakura, D. Coster, R. Doerner, R. Dux, G. Federici, M. Fenstermacher, W. Fundamenski, P. Ghendrih, A. Herrmann, J. Hu, S. Krasheninnikov, G. Kirnev, A. Kreter, V. Kurnaev, B. LaBombard, S. Lisgo, T. Nakano, N. Ohno, H. Pacher, J. Paley, Y. Pan, G. Pautasso, V. Philipps, V. Rohde, D. Rudakov, P. Stangeby, S. Takamura, T. Tanabe, Y. Yang, and S. Zhu, Nuclear Fusion 47, 1189 (2007).

${ }^{3}$ S. J. Zweben and R. W. Gould, Nuclear fusion 25, 171 (1985).

${ }^{4}$ D. A. D'Ippolito, J. R. Myra, and S. J. Zweben, Physics of Plasmas 18, 060501 (2011).

${ }^{5}$ S. I. Krasheninnikov, D. A. D'Ippolito, and J. R. Myra, Journal of Plasma Physics 74 (2008), 10.1017/S0022377807006940.

${ }^{6}$ G. Fuchert, G. Birkenmeier, B. Nold, M. Ramisch, and U. Stroth, Plasma Physics and Controlled Fusion 55, 125002 (2013).

${ }^{7}$ S. Garland, G. Fuchert, M. Ramisch, and T. Hirth, Plasma Physics and Controlled Fusion 58, 044012 (2016).

${ }^{8}$ G. Fuchert, G. Birkenmeier, M. Ramisch, and U. Stroth, Plasma Physics and Controlled Fusion 58, 054005 (2016).

${ }^{9}$ E. J. Kolmes, I. E. Ochs, M. E. Mlodik, J.-M. Rax, R. Gueroult, and N. J. Fisch, Physics of Plasmas 26, 082309 (2019).

${ }^{10}$ N. Bisai, A. Das, S. Deshpande, R. Jha, P. Kaw, A. Sen, and R. Singh, Physics of Plasmas 12, 102515 (2005).

${ }^{11}$ S. Garland, K. Reuther, M. Ramisch, and P. Manz, Physics of Plasmas 24, 112307 (2017).

${ }^{12}$ P. D. Dura, B. Hnat, J. Robinson, and R. O. Dendy, Physics of Plasmas 19, 092301 (2012).

${ }^{13}$ N. Krause, C. Lechte, J. Stöber, U. Stroth, E. Ascasibar, J. Alonso, and S. Niedner, Review of Scientific Instruments 73, 3474 (2002).

${ }^{14}$ A. Hasegawa and M. Wakatani, Physical Review Letters 50, 682 (1983).

${ }^{15}$ M. Wakatani and A. Hasegawa, Physics of Fluids (1958-1988) 27, 611 (1984).

${ }^{16}$ J. M. Dewhurst, B. Hnat, and R. O. Dendy, Physics of Plasmas 16, 072306 (2009).

${ }^{17}$ A. N. Kolmogorov, Proceedings: Mathematical and Physical Sciences 434, 9 (1991).

${ }^{18}$ U. Frisch, Turbulence: The Legacy of A. N. Kolmogorov (Cambridge University Press, Cambridge, 1995).

${ }^{19}$ R. H. Kraichnan, Physics of Fluids 10, 1417 (1967).

${ }^{20}$ R. H. Kraichnan and D. Montgomery, Reports on Progress in Physics 43, 547 (1980).

${ }^{21} \mathrm{~S}$. Pope, Turbulent Flows (Cambridge university press, Cambridge, 2001).

${ }^{22}$ R. T. Cerbus and W. I. Goldburg, Physics of Fluids 25, 105111 (2013), 1306.0595.

${ }^{23}$ F. Anselmet, Y. Gagne, E. J. Hopfinger, and R. A. Antonia, Journal of Fluid Mechanics 140, 63 (1984).

${ }^{24}$ S. Futatani, S. Benkadda, Y. Nakamura, and K. Kondo, Physics of Plasmas 15, 072506 (2008).

${ }^{25}$ G. Y. Antar, P. Devynck, X. Garbet, and S. C. Luckhardt, Physics of Plasmas 8, 1612 (2001).

${ }^{26}$ V. P. Budaev, S. Savin, L. Zelenyi, N. Ohno, S. Takamura, and E. Amata, Plasma Physics and Controlled Fusion 50, 074014 (2008).

${ }^{27}$ R. Benzi, S. Ciliberto, R. Tripiccione, C. Baudet, F. Massaioli, and S. Succi, Physical Review E 48, R29 (1993).

${ }^{28}$ Y. Jun and X. L. Wu, Physical Review E 72 (2005), 10.1103/PhysRevE.72.035302.

${ }^{29}$ J. Paret and P. Tabeling, Physics of Fluids 10, 3126 (1998).

${ }^{30}$ G. I. Taylor, Proc. R. Soc. London, Ser. A 164, 476 (1938).

${ }^{31}$ A. Köhn, G. Birkenmeier, A. Chusov, P. Diez, A. Feuer, U. Höfel, H. Höhnle, E. Holzhauer, W. Kasparek, S. Merli, M. Ramisch, J. Seifert, S. Wolf, and U. Stroth, Plasma Physics and Controlled 
Fusion 55, 014010 (2013)

${ }^{32}$ S. Enge, G. Birkenmeier, P. Manz, M. Ramisch, and U. Stroth, Physical Review Letters 105, (2010).

${ }^{33}$ C. Lechte, S. Niedner, and U. Stroth, 4, 34.1 (2002).

${ }^{34}$ U. Stroth, F. Greiner, C. Lechte, N. Mahdizadeh, K. Rahbarnia, and M. Ramisch, Physics of Plasmas 11, 2558 (2004).

${ }^{35}$ M. Ramisch, F. Greiner, N. Mahdizadeh, K. Rahbarnia, and U. Stroth, Plasma Phys. Control. Fusion 49, 777 (2007).

${ }^{36} \mathrm{~N}$. Mahdizadeh, Investigation of Three-Dimensional Turbulent Structures in the Torsatron TJ-K, Ph.D. thesis, Universität Stuttgart (2007).

${ }^{37}$ N. Mahdizadeh, F. Greiner, M. Ramisch, U. Stroth, W. Guttenfelder, C. Lechte, and K. Rahbarnia, Plasma Phys. Control. Fusion 47, 569 (2005).

${ }^{38}$ V. P. Budaev, N. Ohno, S. Masuzaki, T. Morisaki, A. Komori, and S. Takamura, Nuclear Fusion 48, 024014 (2008).

${ }^{39}$ T. Dudok de Wit, Physical Review E 70, (2004).
${ }^{40}$ G. Boffetta, A. Celani, and M. Vergassola, Physical Review E 61, R29 (2000).

${ }^{41}$ S. J. Camargo, D. Biskamp, and B. D. Scott, Physics of Plasmas 2, 48 (1995).

${ }^{42}$ W. J. T. Bos, B. Kadoch, S. Neffaa, and K. Schneider, Physica D: Nonlinear Phenomena 239, 1269 (2010).

${ }^{43}$ B. Kadoch, W. J. T. Bos, and K. Schneider, Physical Review Letters 105, 145001 (2010).

${ }^{44}$ W. Horton, Physics Reports 192, 1 (1990).

${ }^{45}$ L. Chen, M. Chance, and C. Cheng, Nuclear Fusion 20, 901 (1980).

${ }^{46}$ G. Birkenmeier, M. Ramisch, P. Manz, B. Nold, and U. Stroth, Physical Review Letters 107 (2011), 10.1103/PhysRevLett.107.025001.

${ }^{47}$ M. H. Nasim, T. Rafiq, and M. Persson, Plasma Phys. \& Controlled Fusion 46, 193 (2004).

${ }^{48}$ M. Ramisch, G. Birkenmeier, A. Köhn, and U. Stroth, in Proc. of the 38th EPS Conference on Plasma Physics, Vol. 35, P5.111 (Strasbourg, France, 2011). 